УДК 666. 983

\title{
ИСПОЛЬЗОВАНИЕ СИСТЕМЫ МОДЕЛИРОВАНИЯ ПОТОКОВ ДЛЯ СРАВНЕНИЯ ПРОПУСКНОЙ СПОСОБНОСТИ КЛАПАНОВ, ИСПОЛЬЗУЕМЫХ В КОНСТРУКЦИЯХ ПОРШНЕВЫХ БЕТОНОНАСОСОВ
}

Д-р техн. наук А.Ю. Крот

\section{ВИКОРИСТАННЯ СИСТЕМИ МОДЕЛЮВАННЯ ПОТОКІВ ДЛЯ ПОРІВНЯННЯ ПРОПУСКНОЇ ЗДАТНОСТІ КЛАПАНІВ, ВИКОРИСТОВУВАНИХ У КОНСТРУКЦІЯХ ПОРШНЕВИХ БЕТОНОНАСОСІВ}

Д-р техн. наук О.Ю. Крот

\section{USING FLOW MODELLING FOR COMPARISON CAPACITY VALVE THAT USE IN PISTON CONCRETE PUMPS}

\section{DSc O. Krot}

Рассмотрены неуправляемые клапаны, которые используются в поршневых бетононасосах (шаровые и тарельчатые). Моделирование выполнялось методом конечных элементов уравнениями Навье-Стокса и неразрывности потока. Подтверждена вдвое большая пропускная способность шарового клапана. Скорость вблизи стенки в канале с тарельчатым клапаном втрое больше скорости для шарового. Это вызовет износ стенки тарельчатого клапана. Предложено конструктивное решение уменьшения износа стенки.

Ключевые слова: бетононасос, тарельчатые и шаровые клапаны, метод конечных элементов, Навье-Стокса, моделирование

Розглянуто некеровані клапани, щуо використовуються в поршневих бетононасосах (кульові і тарілчасті). Моделювання виконувалося методом кінцевих елементів рівняннями Навье-Стокса і нерозривності потоку. Підтверджена удвічі більша пропускна здатність кульового клапана. Швидкість поблизу стінки в каналі з тарілчастим клапаном утроє більше швидкості для кульового. Це викликає знос стінки тарілчастого клапана. Запропоновано конструктивне рішення зменшення зносу стінки.

Ключові слова: бетононасос, тарілчасті і кульові клапани, метод кінцевих елементів, Навье-Стокса, моделювання.

Was analyzed variants of valves, which are used in piston concrete pumps. Comparison unguided valve based on the results of simulation of the motion flows in pipes, provided with the valves in open position. Modeling was performed using finite element method for large-scale virtual $3 D$-model using the Navier-Stokes equations and the continuity of flow. It was determined flow rates and pressure in the cross sections at the inlet and outlet channels valves. Was confirmed double the bandwidth of a ball valve plate than with comparable geometric dimensions. Velocity near the wall in the channel with the poppet valve corresponding to three times the speed for the ball valve. This will cause that the pipe wall after the poppet valve is worn out 9 times faster than in the embodiment with a ball valve. Was proposed a constructive solution to reduce wear the walls of the poppet valve.

Keywords: concrete, plate and ball valves, the finite element method, Navier-Stokes equations, modeling.

Введение.

Обязательным компонентом современной стройки является бетононасос. Довольно популярными бетононасосами являются поршневые бетононасосы. Они обладают множеством преимуществ в сравнении с другими, в частности, они способны обеспечивать большое давление в 
напорном трубопроводе. Это преимущество является существенным в высотном строительстве, а также в таких технологиях укладки бетона, как торкретирование.

Постановка проблемы в общем

виде.

Поршневой бетононасос представляет собой один или несколько цилиндрических поршневых нагнетателя с системой подачи в них бетона и системами, предотвращающими обратный ход бетонной смеси при обратном ходе поршня (обычно это клапаны) [1, с.330]. Клапаны могут быть как управляемыми (приводимыми в движение от привода, синхронизированного с движением поршней), так и неуправляемыми. Последние хоть и уступают управляемым из-за меньшего к.п.д. насоса, однако обладают важным преимуществом - простотой изготовления и стоимостью.

\section{Цели и задачи исследований.}

В данной работе два варианта неуправляемых клапана (шаровый и тарельчатый, зафиксированный на шарнирной оси) с целью выявления достоинств и недостатков каждого из них рассмотрены с точки зрения меньшего сопротивления и большей скорости прохождения бетона через открытый клапан. Сравнение клапанов основывалось на результатах моделирования движения потоков в трубах, снабженных этими клапанами в открытом положении. Для создания одинаковых условий при моделировании трубы с клапанами соединены с одним общим источником среды и расположены на равном расстоянии от источника; обе трубы имеют горизонтальное расположение (рис. 1).

\section{Основная часть.}

3D-модель создана в системе Компас3D, экспортирована в файл стереолитографии (*.stl), импортирована в программу анализа потоков. Для моделирования процесса применялась система моделирования движения жидкости и газа Flow Vision, основанная на методе конечных элементов. Версия программы с ограниченной функциональностью распространяется бесплатно. Ограничение функционала проявляется в ограниченном количестве конечных элементов.

Среда моделировалась как несжимаемая жидкость [вода плотностью 1000 кг/м ${ }^{3}$, молекулярная масса 18 а.е.м. (атомных единиц массы, $\mathrm{H}_{2} \mathrm{O} / 1+1+16 /$ ), молекулярная вязкость (коэффициент динамической вязкости при $20^{\circ} \mathrm{C}$ ) $\left.0,001 \Pi a^{*} \mathrm{c}\right]$.

Модель основана на уравнении Навье-Стокса.

$$
\begin{gathered}
\frac{\partial \rho V}{\partial t}+\nabla(\rho V \times V)=-\nabla P+\nabla\left(\left(\mu+\mu_{t}\right)\left(\nabla V+(\nabla V)^{T}\right)\right)+S \\
\frac{\partial \rho}{\partial t}+\nabla(\rho V)=0,
\end{gathered}
$$

где источник $S$ равняется:

$$
\begin{aligned}
S= & \left(\rho-\rho_{\text {hyd }}\right) g+\rho B+R . \\
& \text { Здесь } \rho, \frac{\text { Кг }}{\mathrm{M}^{3}} \text { - плотность; } V, \frac{\mathrm{M}}{\mathrm{c}} \text { - }
\end{aligned}
$$

скорость; $\nabla$ - оператор Гамильтона

(набла оператор); $\quad P$, Па - давление;

$\mu, \frac{\text { кГ }}{\mathrm{M} \cdot \mathrm{c}}$ - вязкость; $\mu_{t}, \frac{\mathrm{\kappa} \Gamma}{\mathrm{M} \cdot \mathrm{c}}$ - турбулентная кинематическая вязкость, $B, \frac{\mathrm{M}}{\mathrm{c}^{2}}$ - составные от сил вращения (Кориолиса и центробежной),

$$
B=-2 \omega \times V-\omega \times \omega \times r ; \omega, \frac{\text { рад }}{\mathrm{c}} \text { - }
$$

угловая скорость; $r, \mathrm{M} \mathrm{-} \mathrm{радиус.}$ 


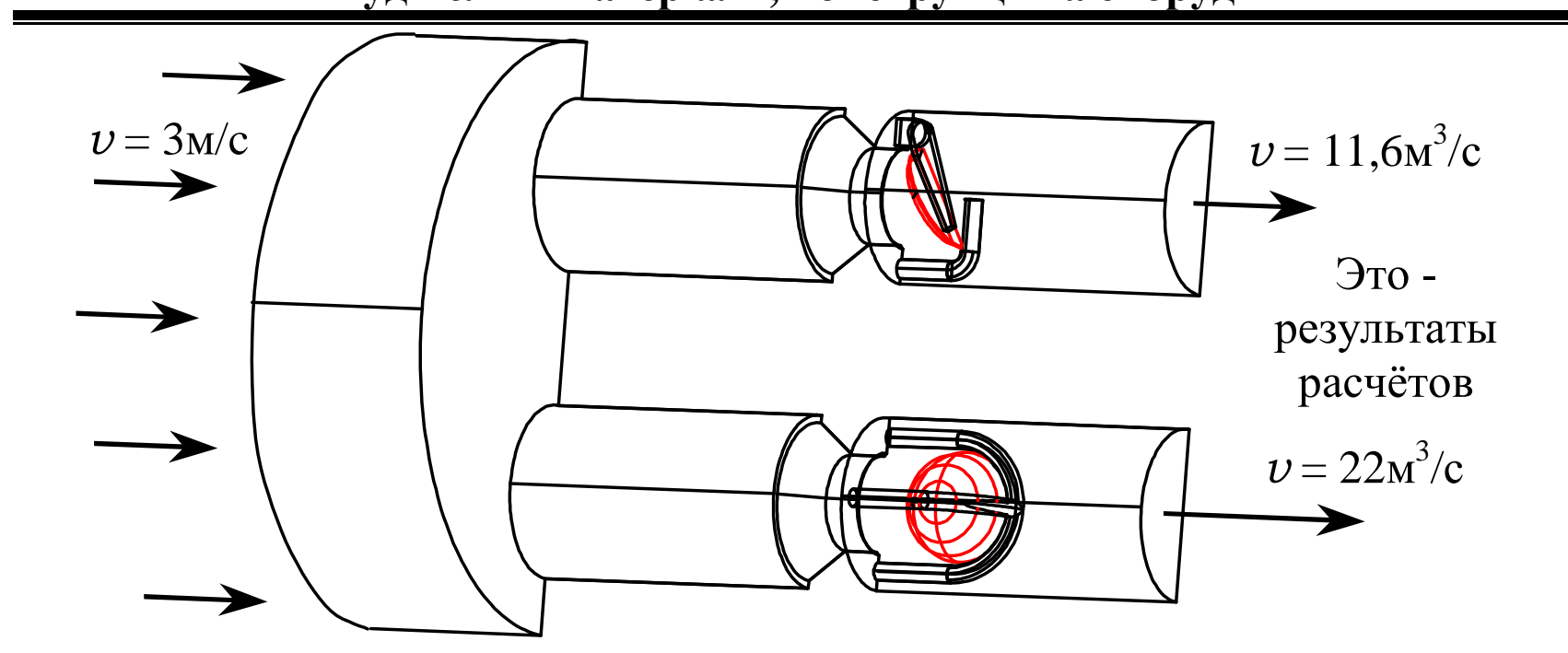

\section{Рис. 1. Схема подключения труб с клапанами к емкости (входу)}

В соответствии с методом конечных элементов, все исследуемое пространство разбивается на определенное количество элементарных участков (рис. 2), в каждом из которых в соответствии с выбранной моделью, свойствами вещества, начальными условиями и др. рассчитывается состояние. К характеристикам состояния каждого элемента относятся давление, скорость (в любом направлении), концентрация, плотность, температура и др. Любая из этих характеристик после процедуры расчета может быть отображена на участках модели в виде графика, заливки или в другом удобном наглядном виде. рис. 3.

Размеры исследуемых клапанов - на

$$
\begin{aligned}
& \text { Проверка адекватности модели } \\
& \text { основана нравнении } \\
& \text { производительности на входе в систему и } \\
& \text { на выходе из неё. } \\
& \text { Средняя скорость на выходе } 3 \mathrm{M} / \mathrm{c} ; \\
& \text { расход } v \cdot \pi \cdot \mathrm{R}^{2}=3 \cdot 3,14 \cdot 0,15^{2}=0,212 \mathrm{M}^{3} / \mathrm{c} \\
& 0,074+0,14=0,214 \mathrm{~m}^{3} / \mathrm{c} \quad\left(\approx 0,212 \mathrm{~m}^{3} / \mathrm{c}\right)
\end{aligned}
$$

основана на сравнении
проверка «сплошности» потока подтверждает адекватность моделирования.

\section{Выводы.}

1. В результате моделирования каналов с двумя открытыми клапанами разного типа подтверждена вдвое большая пропускная способность шарового клапана, чем тарельчатого при сопоставимых геометрических размерах (средняя скорость $22 \mathrm{M} / \mathrm{c}$ у шарового клапана против $11,6 \mathrm{~m} / \mathrm{c}$ у тарельчатого).

2. Скорость вблизи стенки в канале с тарельчатым клапаном втрое больше соответствующей скорости для шарового клапана $[33 \mathrm{M} / \mathrm{c}$ (рис. 4,6$)$ и $11 \mathrm{~m} / \mathrm{c}$ (рис. 4,в)]. Это, очевидно, приведет к тому, что стенка трубы после тарельчатого клапана будет изношена в 9 раз быстрее, чем в варианте с шаровым клапаном (пропорционально кинетической энергии потока).

3. Возможным решением проблемы снижения пропускной способности тарельчатого клапана может быть устройство зоны расширенного сечения канала (устройство расширителя, рис. 5). Эффективность такого решения может быть подтверждено моделированием (моделирование пока не выполнялось). 


\section{Будівельні матеріали, конструкції та споруди}
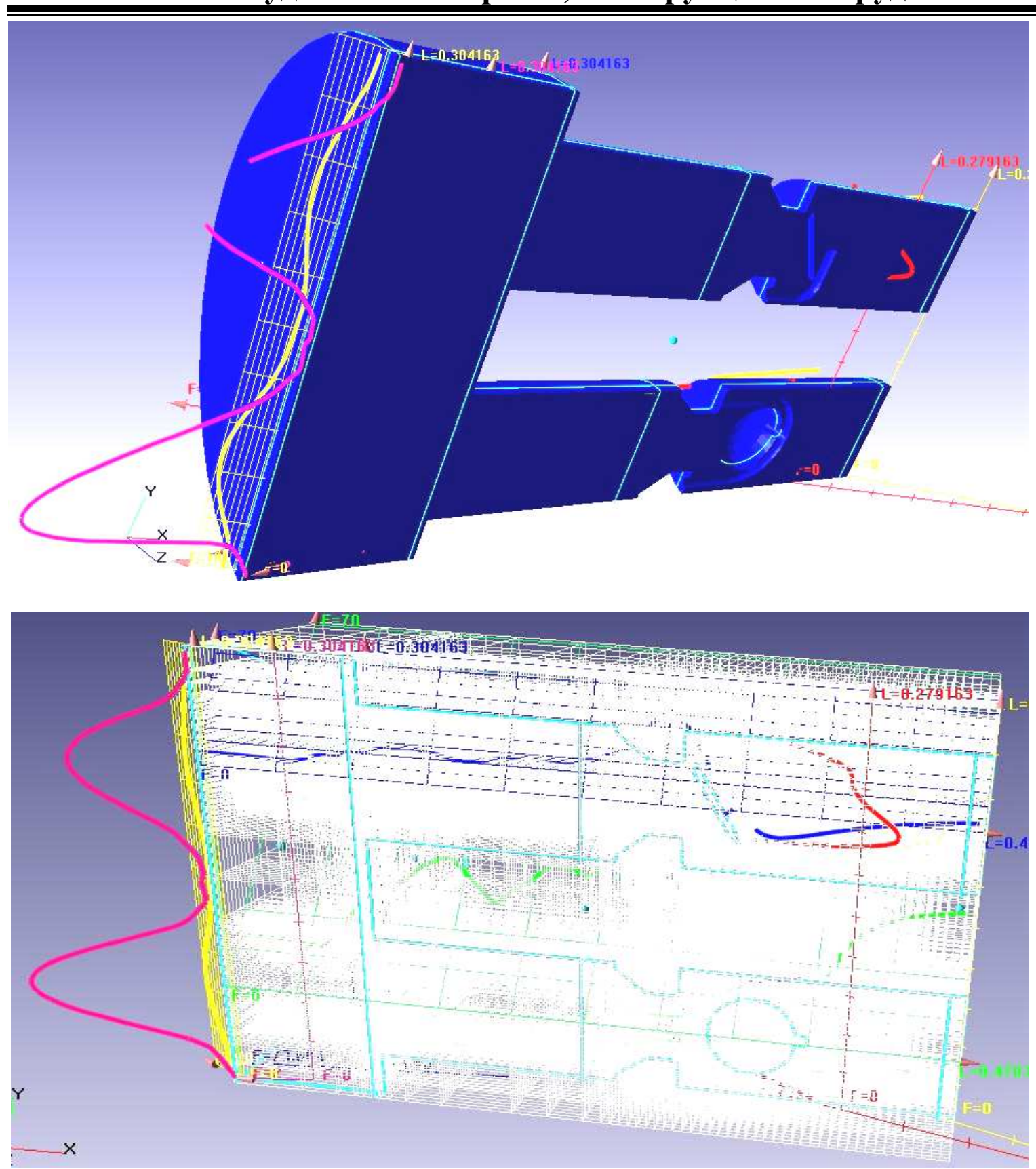

Рис. 2. 3D-модель и расчетная сетка 


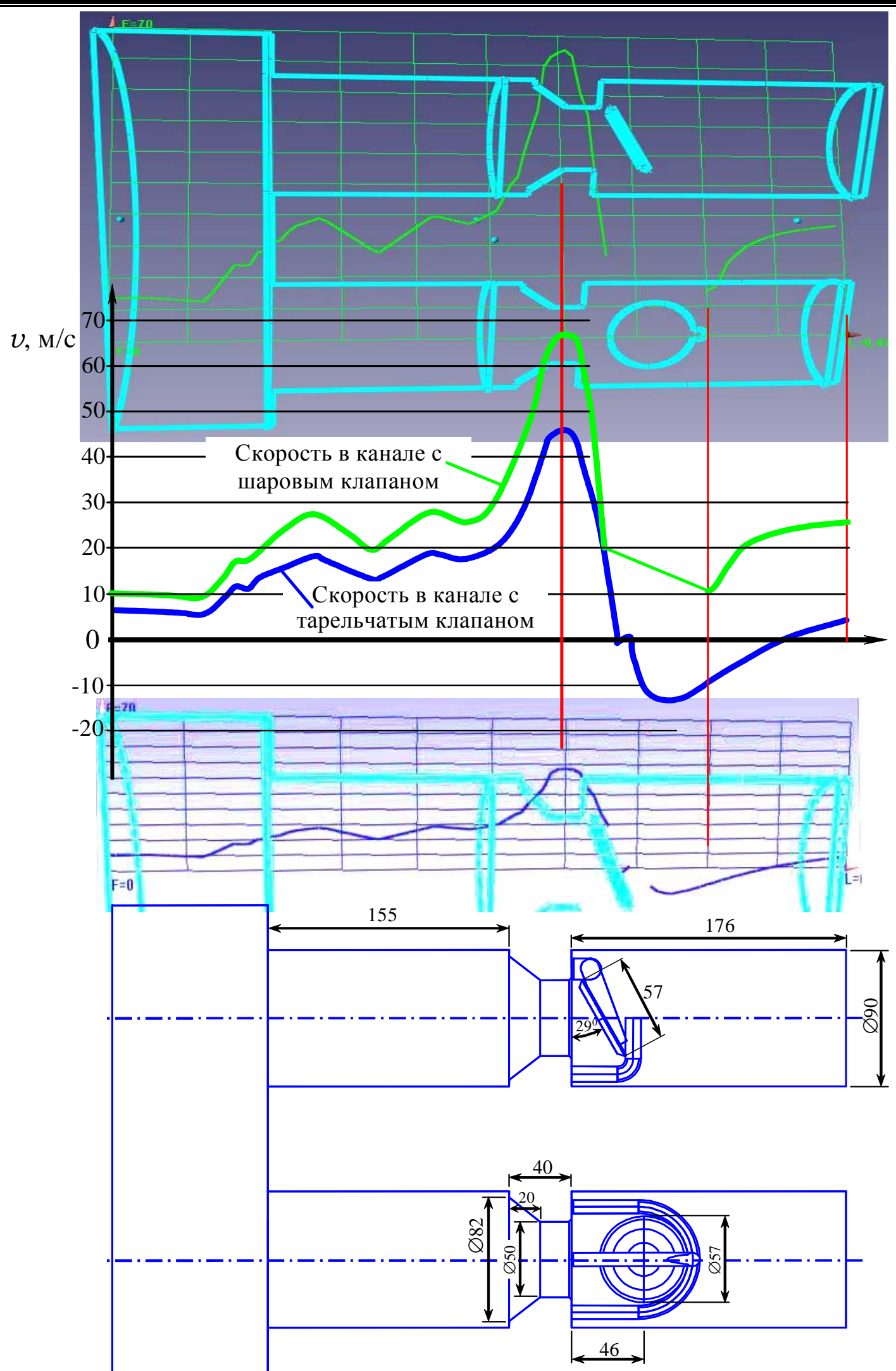

Рис. 3. Эпюры скоростей в каналах с клапанами и размеры клапанов 


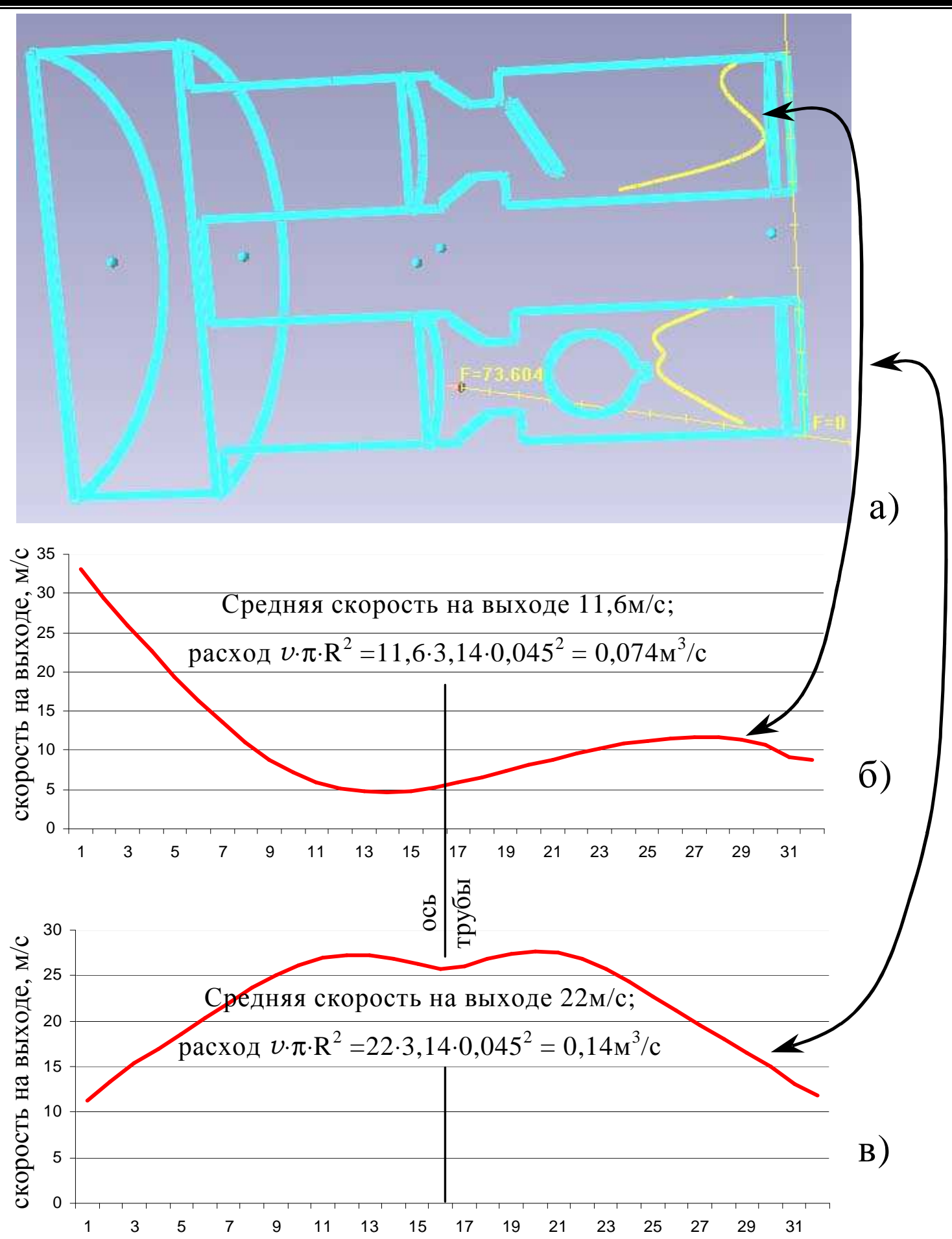

Рис. 4. Скорости потоков на выходе из клапанов:

a) общая схема;

б) тарельчатый клапан;

в) шаровый клапан.

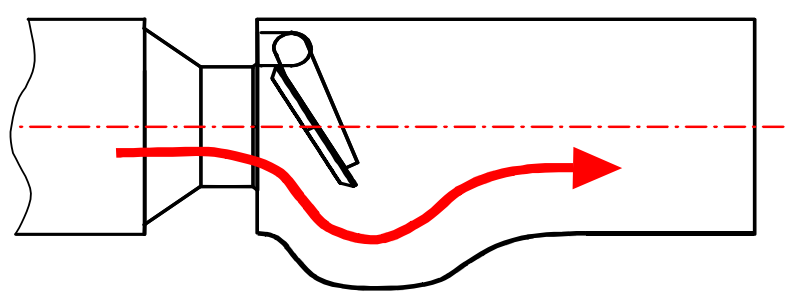

Рис. 5. Расширитель в канале после тарельчатого клапана 


\section{Список использованных источников}

1. Строительные и дорожные машины и основы автоматизации :учебное пособие / И.Ф. Дьяков; /Ульян. гос. техн. ун-т. - Ульяновск : УлГТУ, 2007. - 516 с.

Крот Александр Юльевич - д.т.н., профессор кафедры механизации строительных процессов, Харьковский национальный университет строительства и архитектуры (ХНУСА), Сумская, 40, Харьков, 61002, Украина; tanko_drom@rambler.ru; +38 (057) 7000282.

Krot Oleksandr - Doctor of Technical Sciences, Professor, Department of Mechanization of Construction Processes, Kharkiv National University of Civil Engineering and Architecture (KhNUSA), 40 Sums'ka St., Kahrkiv, 61002, Ukraine; tanko_drom@rambler.ru; +38 (057) 7000282.

Стаття прийнята 05.06.2015 p 